\title{
Encumbrance-Free Telepresence System with Real-Time 3D Capture and Display using Commodity Depth Cameras
}

\author{
Andrew Maimone* Henry Fuchs ${ }^{\dagger}$ \\ Department of Computer Science \\ University of North Carolina at Chapel Hill
}
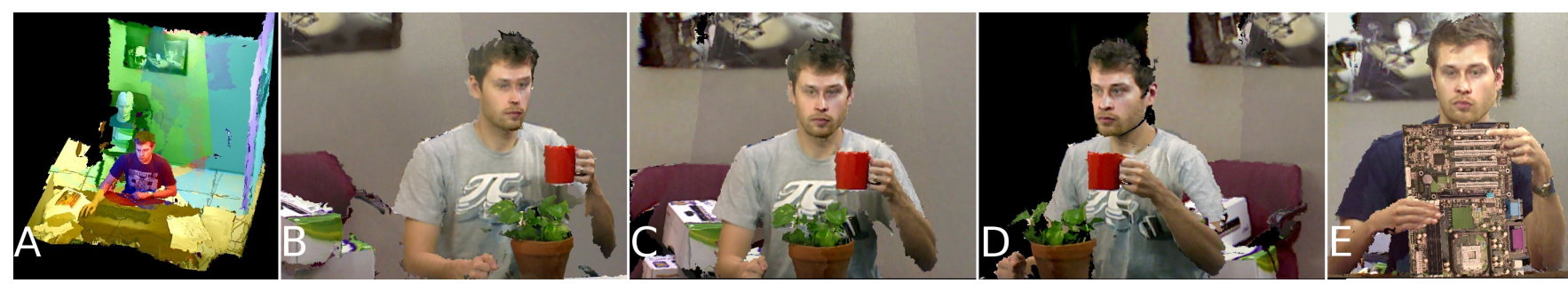

Figure 1: Left to right: A) Camera coverage with five color-coded camera contributions. B-D) Three views of a live capture session. E) A 3D virtual object (circuit board) is incorporated into a live 3D capture session and appropriately occludes real objects.

\begin{abstract}
This paper introduces a proof-of-concept telepresence system that offers fully dynamic, real-time 3D scene capture and continuousviewpoint, head-tracked stereo $3 \mathrm{D}$ display without requiring the user to wear any tracking or viewing apparatus. We present a complete software and hardware framework for implementing the system, which is based on an array of commodity Microsoft Kinect ${ }^{\mathrm{TM}}$ color-plus-depth cameras. Novel contributions include an algorithm for merging data between multiple depth cameras and techniques for automatic color calibration and preserving stereo quality even with low rendering rates. Also presented is a solution to the problem of interference that occurs between Kinect cameras with overlapping views. Emphasis is placed on a fully GPUaccelerated data processing and rendering pipeline that can apply hole filling, smoothing, data merger, surface generation, and color correction at rates of up to 100 million triangles/sec on a single PC and graphics board. Also presented is a Kinect-based markerless tracking system that combines $2 \mathrm{D}$ eye recognition with depth information to allow head-tracked stereo views to be rendered for a parallax barrier autostereoscopic display. Our system is affordable and reproducible, offering the opportunity to easily deliver 3D telepresence beyond the researcher's lab.
\end{abstract}

Keywords: teleconferencing, virtual reality, sensor fusion, camera calibration, color calibration, surface fitting, filtering, parallel processing, computer vision, tracking, object recognition, threedimensional displays

Index Terms: H.4.3 [Information Systems Applications]: Communications Applications-Computer conferencing, teleconferencing, and videoconferencing

\section{INTRODUCTION}

A long-standing goal [21] of telepresence has been to unite distant workspaces through a shared virtual window, allowing remote col-

*e-mail: maimone@cs.unc.edu

†e-mail: fuchs@cs.unc.edu laborators to see into each other's environments as if these were extensions of their own.

In 2002, UNC/UPenn researches created an early realization of this goal by combining a static 3D model of an office with nearreal-time 3D acqusition of a remote user and displaying the result in head-tracked stereo at interactive rates. Since then, several improved 3D capture and display systems have been introduced. In 2004, the MERL 3DTV [14] system offered a glasses and trackerfree capture and display system using an array of 16 cameras and a lenticular autostereo display. However, framerate was low (12 $\mathrm{Hz}$ ) and the number of viewing zones was limited and repeating. In 2008, the Fraunhofer Institute and the Heinrich-Hertz Institute introduced 3DPresence [22], an improved lenticular-display based system. The system supported multiple views for several participants seated around a table, but like in the MERL system, the number of views was limited and only horizontal parallax was available. In 2009, USC ICT researchers presented a telepresence system [9] that used structured light for 3D acquisition and a volumetric 3D display. The system provided real-time capture, nearly continuous points of view and required no tracking markers or glasses, but capture and display were limited to a head-size volume. In 2010, Holografika introduced a compelling system [2] consisting of a large array of projectors and cameras offering fully dynamic real-time 3D capture and tracker-less autostereo display. The system, however, featured only a moderate capture rate $(10-15 \mathrm{~Hz})$ and did not offer fully continuous points of view - interpolation was performed between a linear array of densely placed 2D cameras and only horizontal parallax was provided. Featuring 27 cameras, 3 PCs, and scores of projectors, it was also a very expensive system to build. Also noteworthy are a group of systems $[7,12,1,20]$ with the alternate goal of placing users in a shared virtual space rather than capturing and presenting users within their physical environments.

In this paper, we aim to overcome some of the limitations of previous telepresence systems. Our system is fully dynamic, presenting a live view of remote users as well as their environments, allowing users to enhance communication by utilizing surrounding objects. Continuous viewpoints are supported, allowing users to look around a remote scene from exactly the perspective corresponding to their head position, rather than from a single or set of fixed vantages. This grants users the ability to see around obstructions and gain more information about the remote scene. Gaze is 


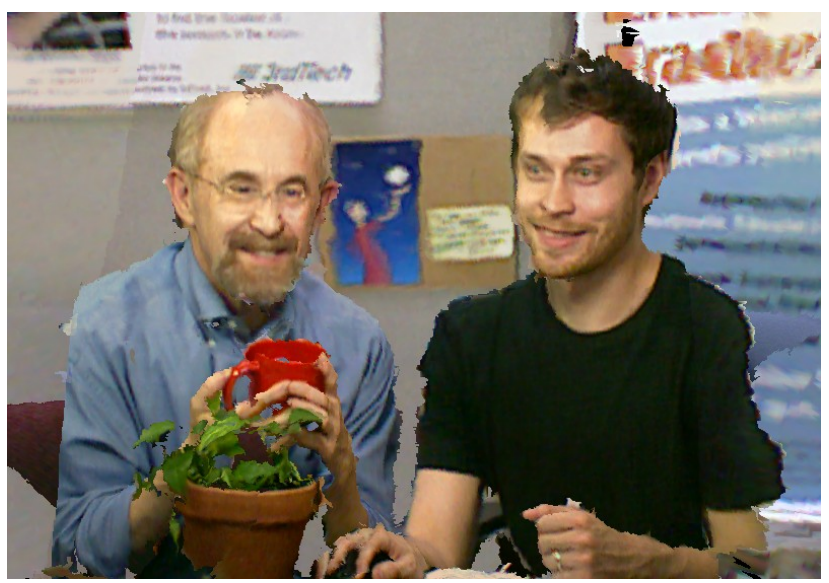

Figure 2: Two users in 3D scene.

preserved, allowing participants to make eye contact; research [18] has shown the absence of correct gaze can cause a loss of nonverbal communication. Stereo views are provided, which have been shown to increase the sense of shared presence [18]. Finally, tracking and viewing apparatuses have been eliminated - 3D glasses obstruct eye contact between participants and shutter glasses have been found to be "disruptive" to over $90 \%$ of users [18]. We believe our system is the first to incorporate all of these characteristics: fully dynamic 3D scene capture, continuous look-around ability with full parallax, gaze preservation, and stereo display without the use of any encumbrances.

\section{BACKGROUND AND CONTRIBUTIONS}

Our system is based on the Microsoft Kinect ${ }^{\mathrm{TM}}$ sensor, a widely available, inexpensive (\$150) device that provides color image, infrared image, depth map, and audio capture. Depth data is acquired using imperceptible structured light techniques; a static dot pattern projected with an IR laser is captured with an IR camera and compared to a known pattern [6]. Depth images are provided at $640 \times 480$ resolution at $30 \mathrm{~Hz}$; color and IR images may be captured at this resolution and rate or at $1280 \times 1024$ and approximately 10 $\mathrm{Hz}$. The unit provides a $58^{\circ} \times 45^{\circ}$ field of view and a depth accuracy rated as $1 \mathrm{~cm}$ at $1 \mathrm{~m}$, with a $0.8 \mathrm{~m}$ to $3.5 \mathrm{~m}$ range ${ }^{1}$. (Our units return depth readings for surfaces as near as $0.5 \mathrm{~m}$.)

Utilizing several strategically placed and calibrated Kinect sensors, there is an opportunity to capture an entire room-sized scene in real-time. This scene could be rendered from exactly the remote user's perspective, providing for correct gaze and continuous viewpoints. Eye position tracking is required to provide for continuous viewpoints; 2D eye detection combined with the Kinect's depth data provides a markerless tracking solution.

However, as a device not designed for general purpose 3D scene capture or tracking, the Kinect presents some challenges for our intended purposes. Since each sensor projects a fixed structured light pattern at roughly the same wavelength, inter-unit interference is a major problem. The device, as controlled with the drivers currently available, provides auto-white balance and exposure that cannot be disabled, presenting difficultly for seamlessly integrating color-matched data between cameras. The capture frame rate, 30 $\mathrm{Hz}$, is suitable for scene acquisition but is inadequate for responsive tracking.

This paper aims to provide solutions to these challenges as well as introduce an eye position tracking system based on the Kinect that is used with an autostereo display. Our specific contributions are as follows:

${ }^{1}$ http: //www.primesense.com/

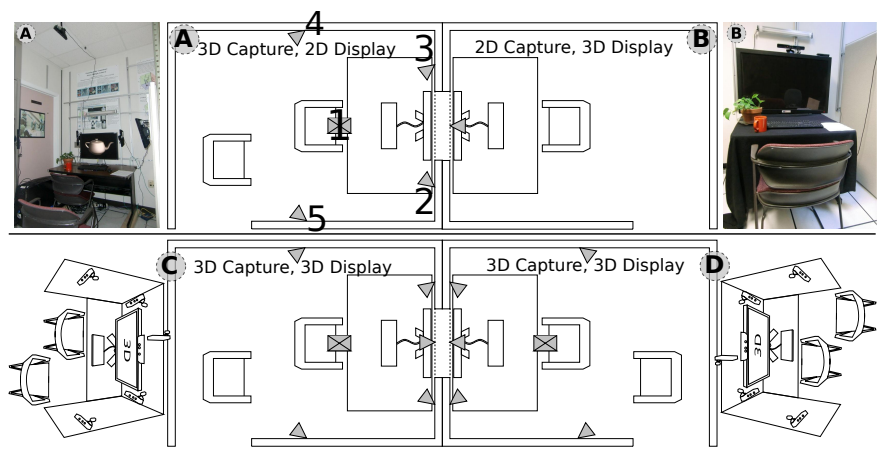

Figure 3: System Layout. Top: Demonstrated proof-of-concept system configuration. Bottom: Proposed ideal configuration.

1. A software solution to the Kinect interference problem that provides hole filling and smoothing

2. A visibility-based algorithm to merge data between cameras

3. A visibility-based method for dynamic color matching between color-plus-depth cameras

4. A system for combining $2 \mathrm{D}$ eye recognition with depth data to provide 3D eye position tracking

5. A technique for preserving high-quality head-tracked stereo viewing on fixed parallax barrier displays even at low rendering rates

We also present a GPU-accelerated software framework that implements hole filling, smoothing, data merging, surface generation, and color correction at interactive rates for five depth cameras on a single PC and graphics board.

\section{System OVERVieW}

\subsection{Physical Layout}

Figure 3 shows the layout of our system. The two spaces are physically separated, but a view of the other space can be seen through the display as if the spaces were aligned with a shared hole in the wall. The bottom of the figure shows our "ideal" configuration - 3D capture and 3D display are supported on both sides (spaces C,D).

The top of Figure 3 shows the actual configuration used for our proof-of-concept system. The system utilizes two office cubicles (approximately $1.9 \mathrm{~m} \times 2.4 \mathrm{~m}$ ). Space A offers 3D capture and 2D display of space B, while space B features $2 \mathrm{D}$ capture and headtracked 3D display of space A. This configuration allowed us to demonstrate 3D capture, 3D display, and eye gaze preservation (for one side) while requiring only the single autostereo display that we had available.

\subsection{Hardware Configuration}

Both spaces in our proof-of-concept system share a single PC with a 4-core Intel Core i7-960 CPU, 6GB of RAM and an Nvidia GeForce GTX 295 graphics board. Six Microsoft Kinect sensors are connected to the PC. The 2D display side features a 30 in LCD monitor, while the 3D display side uses a 40 in X3D Technologies autostereo display.

We avoided networking and audio in our proof-of-concept system since both spaces are run from a single PC and are in close proximity. We plan to address these omissions in a future system.

\subsection{Software Overview}

Operating System and APIs Our test system runs on 64bit Linux (Ubuntu 10.10) and uses the OpenNI ${ }^{2}$ API along with

${ }^{2}$ http: //www.openni.org/ 


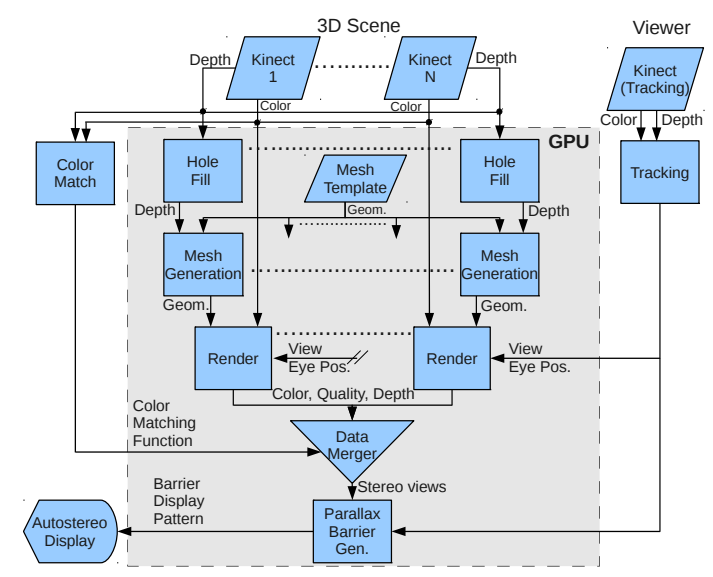

Figure 4: Data Processing and Rendering Pipeline

a Kinect driver ${ }^{3}$ to communicate with the sensors. OpenGL is used for rendering, the OpenGL shader language (GLSL) is used for programmable GPU operations, and GLUT is used for windowing and user input. The OpenCV ${ }^{4}$ computer vision library was utilized for camera calibration and tracking.

Data Processing and Rendering Pipeline The following rendering pipeline is used in our system (Figure 4):

1. When new data is available, read color and depth images from Kinect units and upload to GPU

2. Smooth and fill holes in depth image.

3. For each Kinect's data, form triangle mesh using depth data.

4. For each Kinect's data, apply color texture to triangle mesh and estimate quality at each rendered pixel; render from the tracked user's current position, saving color, quality, and depth values.

5. Merge data for all Kinect units using saved color, quality and depth information.

6. Repeat steps 3-5 for other eye's view

7. Assemble the two stereo viewpoints into pattern required by autostereo display, draw to screen.

8. While next 3D scene is being generated, periodically redraw pattern required by autostereo display using the last rendered frame and the new estimated eye position.

GPU Acceleration Overview To maximize performance, all real-time graphics-related data processing algorithms are performed on the GPU (using the OpenGL Shader Language) to reduce CPU/GPU memory transfer overhead and take advantage of GPU parallelism. CPU/GPU memory transfers are kept to a minimum: the five Kinects' color and depth images are uploaded to the GPU, but no other major data transfers take place.

System Component Rates Since our entire system does not run at the desirable rate of $\geq 60 \mathrm{~Hz}$ on our current hardware, we allow three rates in our system to run asynchronously to allow for interactive rendering and high stereo quality. The first is the reconstruction rate, the rate at which new data is incorporated into the rendered scene, which involves uploading new color and depth data to the GPU, hole filling, smoothing, and surface generation. The second rate is the rendering rate, the pace at which the scene is rendered from a new viewing perspective. In our system, this also includes our data merger algorithm, which is visibility-based. The

\footnotetext{
${ }^{3}$ https://github.com/avin2/Sensorkinect

${ }^{4}$ http://opencv.willowgarage.com/
}

final rate is the parallax barrier pattern generation rate, the rate at which new patterns are generated for our autostereo display from new estimated eye positions.

The independence of the reconstruction rate from the rendering rate helps to keep the system running at interactive rates as more cameras are added to the system; a study by Meehan [16] found a positive correlation between framerate and sense of presence as the former was increased from 10 to $30 \mathrm{fps}$. The independent parallax barrier pattern generation rate preserves stereo quality during head motion even if the rendering rate decreases. (See Section 5.3.)

Tracking We combine 2D eye detection, depth data, and motion tracking to create an unencumbered 3D eye position tracker. Initial eye detection is performed on a color image and eyes are then tracked using pattern matching. Once the $2 \mathrm{D}$ eye position is obtained, Kinect depth data is used to transform the position into 3D. A Kalman filter is used to improve accuracy and interpolate the position of the eyes between sensor updates.

\section{IMPLEMENTATION}

\subsection{Camera Placement, Calibration, and Error Measure- ment}

Camera Placement When placing cameras as shown in the top left of Figure 3, the following factors were considered:

1. Coverage: for our application, coverage is only necessary for surfaces that can be seen by the remote user.

2. Redundancy: Redundant coverage allows preservation of surfaces that are occluded from the viewpoint of a single depth camera, but are still visible by the remote user (e.g. an occluded chest behind a raised hand).

3. Resolution and Accuracy: the resolution available varies with angle and distance (discussion in Section 4.4).

4. Kinect Depth Range: approximately 0.5 m-3.5 m.

5. Kinect Depth Interference: discussion in Section 4.2.

6. Kinect Depth Error: perceived depth error is reduced if camera is near line of sight of user

Camera Calibration To calibrate the Kinect sensors, we used the OpenCV camera calibration routines, which are based on Zhang's method [26]. The routines compute camera intrinsic parameters (focal length, center of projection, radial and tangential distortion coefficients) from two or more images of a detected planar pattern, taken from different orientations. Extrinsic parameters (relative positions and orientations) between two cameras are computed using one or more pairs of images of a detected pattern seen from each camera, along with the intrinsic parameters. Since the Kinect driver is able to register the depth image to the color image, only calibration of the color camera is necessary.

For our test system, camera intrinsics and extrinsics were computed using a checkerboard pattern. For extrinsic computation, we calibrate each camera to a master ceiling-mounted camera, whose viewing frustum conveniently overlaps the frustum of each of the other cameras.

Depth Error Measurement Since our mesh generation and data merger techniques rely on knowledge of the relationship between the distance of a surface from a Kinect depth camera and measurement error, it is beneficial to characterize this relationship. We expect the depth resolution to fall off quadratically with distance.

To verify this, we positioned a planar target parallel to the IR camera's image plane and recorded a $100 \times 100$ grid of depth measurements at the center of the depth image. We performed this experiment at distances of $0.5 \mathrm{~m}$ (device minimum range) to $3.0 \mathrm{~m}$ (beyond maximum range used in our system) at intervals of $0.5 \mathrm{~m}$. 


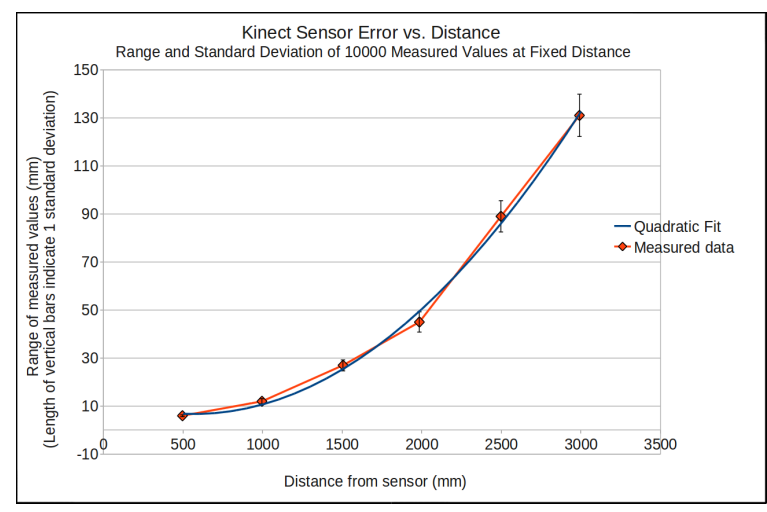

Figure 5: Kinect depth sensor precision with distance. Measured values show quadratic relationship between the distance to the depth camera and the range and standard deviation of depth values.

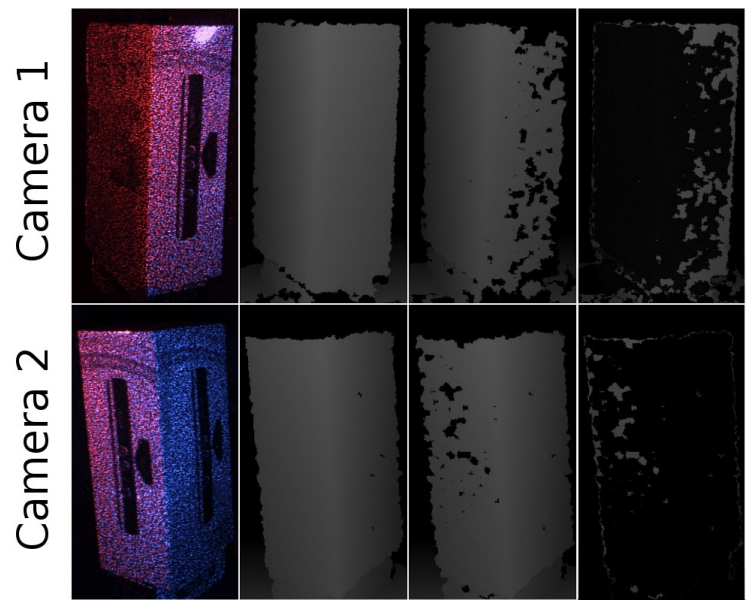

Figure 6: Kinect interference problem. First column: IR images showing combined projected dot pattern from camera 1 (red dots) and camera 2 (blue dots). Second column: depth images with no interference. Third column: depth images with interference from other camera. Fourth column: Difference of second and third columns.

Figure 5 shows the min-max range and standard deviation at each test distance from $0.5 \mathrm{~m}$ to $3.0 \mathrm{~m}$, fitting closely to a quadratic falloff.

\subsection{Multi-Kinect Interference Problem and Solution}

The Multi-Kinect Interference Problem Since each Kinect unit projects the same dot pattern at the same wavelength, each Kinect unit is able to see the projected patterns of all other units and may have trouble distinguishing other units' patterns from its own.

This problem is illustrated in Figure 6. A box was placed near the minimum depth range $(0.6 \mathrm{~m})$ of two Kinect units; their projected patterns overlap prominently and cause interference. Although significant interference is shown in the third column of the figure (there many small areas of missing data, or "holes"), we find several promising aspects of these results. The difference between the depth image with and without interference corresponds mostly to the missing data, not differences in depth values; one needs primarily to fill missing points rather than correct erroneous depth values. Additionally, one can see in the third column of the figure that the missing data varies between depth cameras - to some extent, redundant coverage between units allows depth cameras to fill in each other's holes.
Hardware Solutions We considered, but rejected several hardware solutions to the multi-Kinect interference problem. We contemplated installing a set of alternating synchronized shutters over each unit's IR projector and camera so that each unit would see only its own dot pattern. A serious disadvantage of this approach is that it would reduce frame rate or reduce the light available to the IR camera, depending on how the shutters are used. Another technique considered, but also ruled out, was IR filtering. We measured a group of eight Kinect units with a spectrometer and found that the peak-to-peak range of wavelengths was $2.6 \mathrm{~nm}$, which we found too close to filter practically.

Software Solutions As we did not find a suitable hardware solution to the Kinect interference problem, we looked to software solutions. As mentioned, it is fortunate that the Kinect generally returns no data rather than erroneous data when interference occurs. However, there are other situations in which the sensor returns no depth data. Due to the offset between the IR projector and camera, there are typically some surfaces "in shadow" that can be seen by the camera but receive no projected pattern from the IR laser due to occlusion. Additionally, surfaces may not be seen by the depth camera if they reflect little infrared light or are highly specular. An effective software solution should be able to fill small holes (making the assumption that they are part of a continuous surface), while ignoring large missing surfaces. We hope that the missing large surfaces are captured by another camera that observes the surface from a different location. Also, Kinect interference causes a small amount of high frequency depth noise that should be smoothed.

Hole Filling and Smoothing We aimed for a solution that fills small holes and smoothes depth, but leaves alone large missing surfaces. The obvious starting point for such an approach is a simple smoothing filter (such as Gaussian, box, median, or bilateral), but our application induces additional requirements:

1. Measurement preservation: we do not want to introduce new depth values. Naive smoothing could result in depth values floating in space.

2. Edge preservation: our depth image is aligned to a color texture, so color and depth edges should coincide. A small depth edge shift could cause a texture to be assigned to a physically distant surface. Depth edges at missing data boundaries must be preserved or geometry may expand or contract.

3. Scale independence: from observation, depth noise appears at a higher spatial frequency than holes. Smoothing should take place on a smaller scale than hole filling.

A standard median filter meets some of these requirements (measurement preservation and edge preservation - although not pixelexact). We devised a fast modified median filter (Algorithm 1) that is effective at hole filling while supporting the requirements above.

To allow for scale independence, a two-pass approach is used. In the first pass, an expanded filtering window is used to fill larger holes, but no smoothing is applied (i.e. only missing values are modified). In the second pass, a smaller window is to used to fill any remaining small holes and smooth the depth image. This method is similar to that used in [17], but we use different criteria to determine when the filter is applied.

To ensure that edges are preserved precisely and non-holes are ignored, we apply three constraints to the filtering window: a minimum amount of valid data must be present $\left(t_{c}\right)$, a minimum amount of data must be present at the window edges $\left(t_{e}\right)$, and the range of values in the window must be within a threshold $\left(t_{r}\right)$. At each pixel, if the window constraints are not met, the pixel is left unmodified. These thresholds and heuristics were determined by applying a conventional median filter to sample depth data and inspecting cases that did not meet our requirements listed above. 


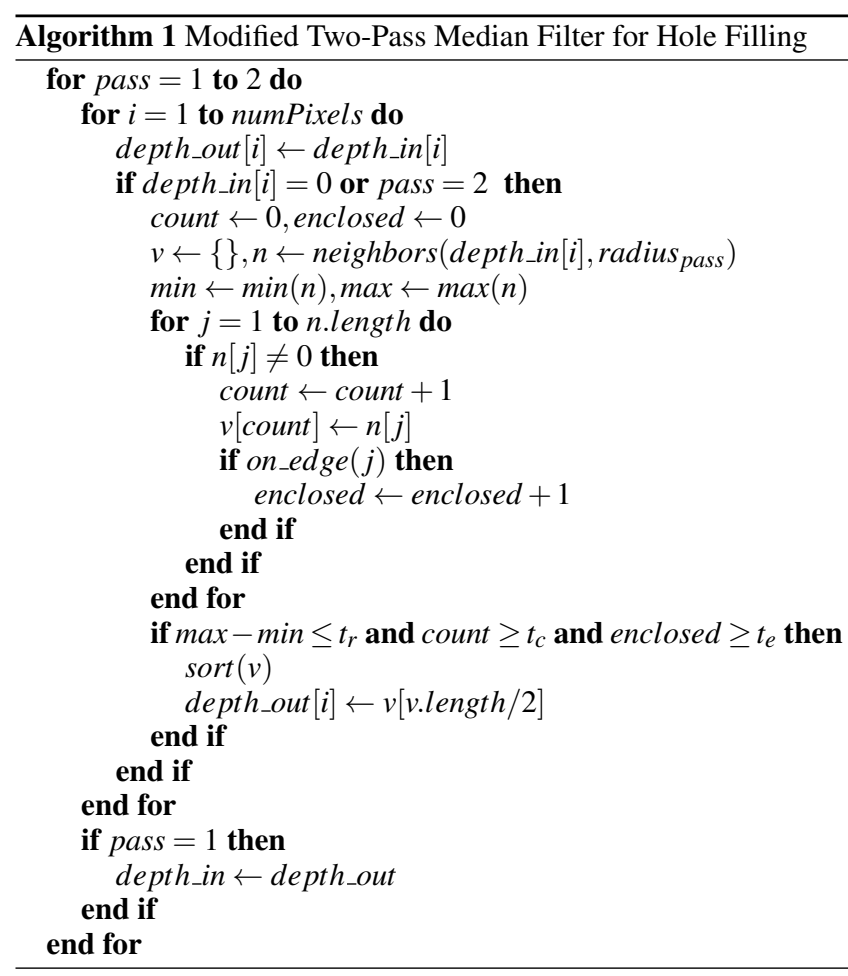

GPU Implementation Our enhanced median filter implementation is based on a conventional median filter implementation by McGuire [15], which uses a branchless hardcoded selection algorithm to obtain the median for fixed radii. To provide high performance for larger radii, we find the approximate median by sampling over the filtering window. The median filter is written as a fragment shader in the OpenGL Shading Language, using textures to exchange data between passes.

\subsection{Mesh Generation}

The Kinect provides per-pixel depth readings that are generally too sparse to render directly as small fixed-size points. Therefore it is useful to create a surface representation using the depth data. Our requirements for surface generation are as follows:

1. Must be continuous if physical surface is continuous

2. Must work in situations with missing data, as is common with Kinect

3. Must detect and preserve depth discontinuities at edges

4. Must be fast ( 5 Kinects generate $>45 \mathrm{M}$ depth readings/sec)

Although approaches exist [10] for directly rendering points from multiple depth images, we chose a triangle mesh surface representation as it meets these requirements and is also supported natively by graphics hardware. We use a simple meshing technique which is described in Algorithm 2. The depth values from the Kinect sensor are used to extrude vertices from a template triangle mesh. Any triangle associated with a vertex that corresponds to a missing depth value is rejected, as are those with a pair of vertices that exceeds a maximum depth threshold. Since the Kinect provides depth data that varies in accuracy with depth, our depth threshold varies with depth as well.

GPU Implementation Our implementation of the simple mesh algorithm takes advantage of the connectivity of a depth image, requiring no geometry to be transferred to the GPU after program initialization. At program start we generate a triangulated plane at the Kinect's depth resolution and store it in GPU memory.

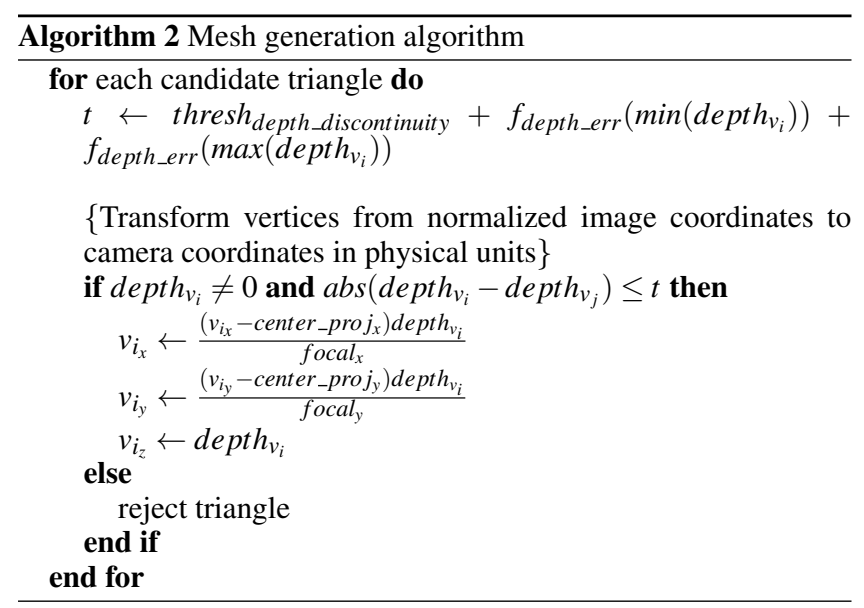

For each new frame, a vertex shader shapes the template plane using camera intrinsics and Kinect depth values, which are accessed through a texture map. A geometry shader is used to reject triangles corresponding to missing depth values or discontinuous surfaces as described in Algorithm 2.

This approach is very bandwidth-efficient - it requires only 16 bits of depth information for each pair of triangles generated and uses the depth map already transferred to GPU memory for the hole filling process. The approach is also fast as all vertex positions are generated on the GPU in parallel.

\subsection{Data Merger}

Overview A goal of our system is to provide coverage of all surfaces that can be seen from the perspective of a remote user. A single Kinect is not able to provide adequate coverage and therefore a means to merge data between multiple units is necessary. When generating meshes we did not discuss a means to merge overlapping surfaces geometrically. Approaches used in stereo vision, such as the visibility-based depth image fusion method of Merrell et al. [17], generally assume high levels of error and inconsistencies (outliers) between maps that must be resolved. The Kinect's structured-light based depth readings, however, are generally free of such outliers and have a low error at near range. In our application, Kinect sensors are used at close proximity and we expect lower and predictable error based on the angle and distance to the camera and measured calibration error. Therefore, we assume that the surfaces have enough fidelity that we can simply draw them on top of each other, avoiding the need for a geometric merger algorithm. This has several performance advantages: the computational expense of performing the merge is spared, runtime varies linearly with the number of cameras, surfaces for all cameras can be processed in parallel, and a fast mesh generation technique (Section 4.3) can be used.

However, even though geometry is sufficiently accurate for our purposes, texture image quality may be poor. Z-fighting between overlapping surfaces with textures that vary in resolution, color balance, and alignment yields unpleasing results. Ideally, we want to utilize only the data from the camera with the highest resolution depth and color information available at a given surface, with a seamless transition to data from adjacent cameras.

Our approach addresses the problem of data merger in image space using a visibility-based approach. The data from each camera is rendered independently for the desired viewpoint, and color information is saved along with a depth and a quality estimate at each pixel. When renderings for all cameras are complete, the depth values are used to determine which cameras can see the front surface. At each pixel, the color values of cameras with a view of the front surface are weighted by the quality estimates. 
Texture Quality and Depth Error Estimation Since our approach relies on the notion of a "quality" measurement at each pixel, we provide an estimate based on resolution - the area on the image sensor available to determine the pixel's color or position. The area is estimated using the cosine of the angle between the surface normal of the pixel and the squared distance from the pixel to the image sensor. The relationship between area and resolution is straightforward for a color image, and we saw previously that the Kinect depth error increases quadratically. We approximate quality by assuming that both color and depth error increase quadratically, yielding the quality value in Equation 1.

$$
\text { quality }=\left(\frac{\cos \theta_{\text {normal } \rightarrow \text { camera }}}{\text { distance }^{2}}\right)^{2}
$$

Note that this formulation is similar to a diffuse lighting calculation with attenuation (for a light positioned at the sensor's location) that can rapidly be performed on almost any graphics hardware.

Our approach also requires determination of which pixels represent the closest surface with respect to viewing position. We store the depth values at each pixel, but due to calibration and depth sensor error the values corresponding to the front surface do not coincide exactly. Equation 2 is used to estimate the range of each depth position, so that the range of depths corresponding to the front surface can be determined.

$$
\left[-e r r_{\text {calib }}-f_{\text {depth_err }}(\text { depth }), e r r_{\text {calib }}+f_{\text {depth_err }}(\text { depth })\right]
$$

Calibration error $\left(e r r_{c a l i b}\right)$ can be estimated using the reprojection error that is returned by the camera calibration routine. Depth error $\left(f_{\text {depth_err }}\right)$ can be estimated using the data from Figure 5 .

Data Merger Algorithm Algorithm 3 describes the process of merging the renderings for each camera. At each pixel, the front surface tolerance is determined by finding the closest depth value that represents the far end of any pixel's estimated depth range. The color values for all pixels with this depth value or nearer are weighted by quality to obtain the final pixel color.

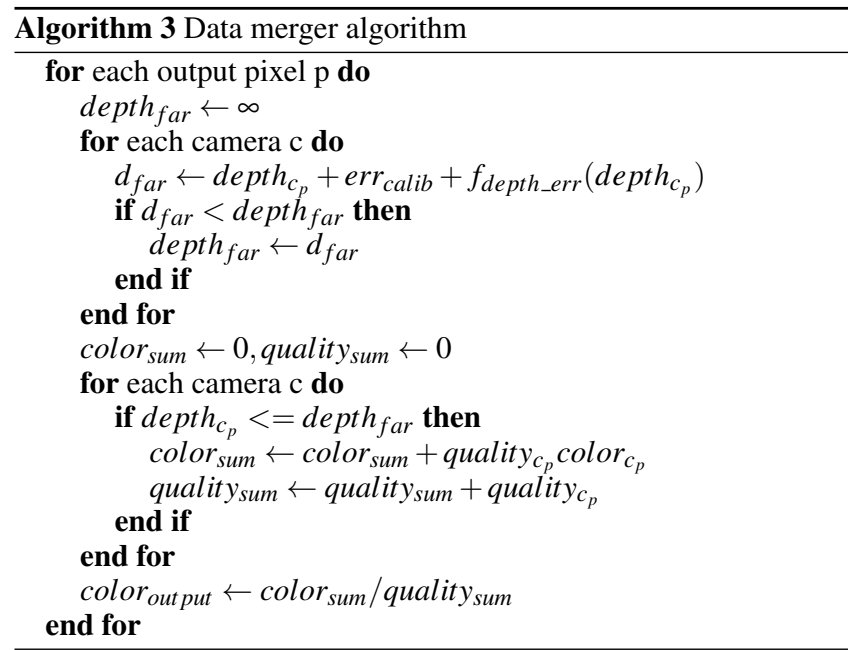

GPU Implementation Our fast GPU implementation supports calculation of depth, quality, and color values in one pass per camera and allows all cameras' renderings to be merged at once in a second pass. When generating the triangle mesh in an OpenGL geometry shader, we compute the distance to the camera and the angle between the camera and surface normal and save these values as vertex attributes. During rasterization, an OpenGL fragment shader computes a color value and a quality value (using Equation 1) at each pixel, storing the quality value in the alpha channel and the depth value from the Z-buffer in a separate texture. When the renderings for all cameras are complete, all data is merged in an OpenGL fragment shader according to Algorithm 3.

\subsection{Multiple Camera Color Matching}

Overview The need for color matching is common for many camera systems, as even the same model device may exhibit different color gamuts [8]. This need is exacerbated in inexpensive devices like the Kinect sensor, which allows only automatic color and exposure control (with present drivers), yielding color values that may vary dramatically between adjacent cameras. Here traditional color matching techniques, such as adjusting color to match a physical target seen by each camera, are ineffective because automatic control may alter color balances at any time. We present an automatic color matching technique that uses depth information to find color correspondences between cameras, which can be used to build a color matching function. We believe this technique may be useful when manual color adjustment is unavailable, or as a fast approximate alternative to conventional matching techniques.

Obtaining Color Correspondences To build a set of color correspondences between cameras, we first find pairs of points from two cameras that correspond to approximately the same point in $3 \mathrm{D}$ space. We assume that each pair of points represents the same point on a diffuse surface in physical space, and therefore should agree in color. To find these point correspondences, we refer to our previously described visibility-based data merger algorithm. The algorithm rendered the scene individually for each Kinect camera and examined corresponding depth values to determine which represented the front surface. For color matching, if two cameras have depth values that represent the front surface at a given pixel, we add their color values to a list of correspondences.

Since this approach is visibility-based, the color correspondences obtained are sensitive to the position of the virtual camera. If the same virtual camera position is used for color matching and rendering, color matching is tailored to the colors actually seen by the user. However, if a pair of cameras have few surfaces in common from the viewpoint used for rendering, or if these surfaces have a limited range of colors, there many be too few correspondences to build a robust color matching function. In this case, point correspondences can be computed from a reference view (such as a bird's eye view), rather than from the view used for rendering. To build more robust color correspondences, additional techniques could be used. For example, the color correspondences could be built from renderings from several viewpoints, or could be collected over time.

Building a Color Matching Function There are many advanced techniques for building color matching functions from a set of color correspondences, such as that of Ilie and Welsh [8]. To demonstrate our approach, we used a simple method - color correspondences were fit to a linear model. Since our color correspondences were noisy (small errors in surface position may result in a large difference in color), we used the RANSAC [5] method for fitting, which is robust to outliers. Figure 7 shows a plot of actual color correspondences (for one channel) and the fitted linear color matching function.

Implementation For our test setup, we matched the colors of each camera to our ceiling-mounted master camera. We elected not to run the color matching function on every frame, as small variations in color matching functions resulted in a color cycling effect. Instead a new color matching function was built whenever the user pressed a function key. As real-time performance was not needed, we implemented color matching functionality on the CPU. We believe our implementation could be improved by performing bundle adjustment across cameras and by running the color matching func- 


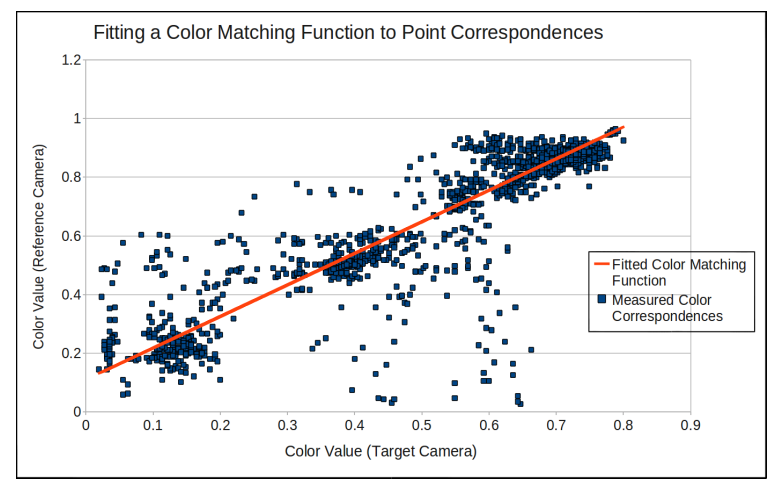

Figure 7: Color matching using 3D point correspondences. Plot shows color correspondences between a pair of cameras for one color channel and the RANSAC-fitted linear color matching function.

tion automatically when some criteria is met.

\subsection{Eye Position Tracking}

Overview To allow our system to render a set of correct stereo viewpoints from the user's position, we need to obtain the position of the viewer's eyes in 3D space. Many approaches to tracking have been devised, such as measuring the magnetic field around a marker, segmenting and triangulating the position of reflective markers as seen by an array of cameras, and using computer vision techniques to recognize objects in images. The latter approach has been used to obtain the 3D positions of eyes with a conventional 2D camera, but assumptions or measurements must be made of the face. We aim to improve these techniques by incorporating depth information. One impressive recent approach [3] used depth information to build a deformable mesh that was tracked to a user's face in real-time, but required a 6.7 second initialization time and achieved only moderate real-time performance $(10-12 \mathrm{~Hz})$. Since we require higher performance and do not need tracking of the entire face, we look to an alternate approach - performing 2D eye detection and transforming the detected position into 3D using the Kinect's depth data.

Requirements Our tracking system should meet the following requirements for use in our telepresence system:

1. Accuracy: at a $1 \mathrm{~m}$ distance, $15 \mathrm{~mm}$ of lateral movement causes the eye to sweep over one display subpixel seen through the barrier of our autostereo display; for best quality results tracking accuracy should be $\pm 7.5 \mathrm{~mm}$.

2. Speed, Latency: we do not anticipate rapid head movements in our application. To support the modest movement of 25 $\mathrm{cm} / \mathrm{sec}$, framerate must be $>33.3 \mathrm{~Hz}$ and latency must be $<30 \mathrm{~ms}$ to meet the accuracy requirements above.

2D Eye Tracking To perform 2D eye detection on the color image, we use Viola [24] and Lienhart's [13] approach of boosted Haar classifiers, as implemented in OpenCV. First the face is detected (using a classifier from Leinhart), and then eyes are detected in the facial region (using classifiers from Castrillon [4]). Once the eyes are found, their pattern is saved and subsequent eye searches are performed using normalized cross correlation. An image pyramid is used to accelerate the cross correlation search. If the strongest response to cross correlation falls below a threshold, detectors are again used to locate facial features. The face is first searched for in the region surrounding the last known eye position; if not found the entire image is again searched. All detection and tracking operations were performed in the CPU, as it was not heavily utilized elsewhere in our system. A single Kinect unit, mounted above the autostereo display, was used for tracking.
Using Depth to Obtain 3D Eye Position Once the center of both eyes have been detected, the $2 \mathrm{D}$ position is transformed into 3D using the Kinect's depth information and measured camera intrinsics and extrinsics. To reduce the effects of noise and missing data, depth values are averaged over a small radius around the eye position. A Kalman filter was used to improve the accuracy and stability of the 3D tracked eye positions as well as predict the locations of the eyes between sensor readings. Although our tracking system requires no prior measurements of the user's face, accuracy can be improved if the true interpupillary distance (IPD) is known. If the system is utilized by a single user over a capture session, an accurate IPD estimate can be learned over time.

Discussion Our tracking system offers several advantages over existing systems. It uses inexpensive hardware (the Kinect sensor) and allows the same device to be used for both tracking and 3D capture at the same time. Since the eyes are tracked independently, our system allows correct calculation of 3D eye positions without measurements or assumptions of face size or IPD.

We believe our system could be improved with a more robust set of feature detectors - our current system allows for only moderate head rotations and does not work well with glasses. Depth data could also be further utilized to improve speed; for example, the face search area could be restricted to depths that are within the range of a seated user. Multiple cameras could be utilized to offer better coverage of a rotated head or to improve the accuracy of the system.

\subsection{Stereo Display}

Overview As mentioned, research [18] has shown that stereo displays can increase the sense of shared presence, although systems requiring 3D glasses obstruct eye contract and have been found to be disruptive to most users. Therefore, we want an autostereo display for our system.

Display Selection Our display system should meet the following requirements for use in our telepresence system:

1. Preservation of full captured color and detail.

2. Large enough to allow remote scene to be observed as lifesized at proper viewing distance.

3. Support for continuous viewpoints and horizontal and vertical parallax.

4. Support for a range of movement typical of a seated user.

5. Interactive update rates that meet our tracking requirements.

We were in possession of a fixed parallax barrier display that met these requirements - an X3D-40 display by X3D technologies (circa 2004). The display measures 40 in diagonally and has a $1280 \times 768$ pixel resolution and a $60 \mathrm{~Hz}$ update rate. Since the display supports only a limited number of views, tracking was employed. In a future system, we intend to utilize a display that supports multiple users, such as the Random Hole display of Ye et al [25].

Rendering for the Display Since our system uses head tracking, we rendered views for the display using off-axis frustra between the eyes and the display. The position of the eyes was determined using the tracking system, and the position of the monitor was measured using our 3D capture system. An OpenGL fragment shader was used to generate the diagonally interleaved pattern needed by our parallax barrier display for each pair of stereo views.

Tracking Problem and Intra-Frame Rendering While using our fixed parallax barrier display, a user may see an incorrect view or significant artifacts (dark black bands or fuzziness) if out of the expected viewing position. If the rendering update rate is lower than the rate required by our tracking system, a user may experience these effects if moving, resulting in poor stereo perception. 
This problem has been addressed previously for dynamic barrier displays [19] by generating the parallax barrier stripes asynchronously at higher rates than rendering takes place. For fixed barrier displays, we developed a new technique to address this problem - rendering barrier display patterns at a higher rate while new frames are rendered more slowly offscreen.

Since the time it takes to generate a parallax barrier pattern for a new eye position is very short and fixed with our implementation, we can draw one or more new barrier patterns while in the process of rendering a frame for the next viewing perspective. These intraframe barrier patterns use the new estimated eye position and the last rendered viewing position, saved in textures. Using OpenGL, we are able to draw to the screen mid-frame by switching between multiple frame buffers. To keep our parallax barrier generation rate and rendering rate independent, we stop to draw a new barrier pattern whenever a fixed amount of time has elapsed during rendering, periodically flushing the pipeline to allow for better time granularity between asynchronous GL calls. The result is a high fixed barrier display rate, independent of rendering rate, at the expense of a small decrease in rendering rate. Specific rates are listed in Section 5.4

\section{Results}

\subsection{Camera Coverage and Calibration Results}

Camera Coverage Our camera arrangement (shown in upper left of Figure 3), includes most of the surfaces seen by a seated remote user, as shown in Figure 1A. Overlapping camera coverage preserves large surfaces on the rear wall, which would otherwise be occluded by the seated user. Redundant coverage also helps prevent self shadowing. For example, in Figures 1B-1D the coffee cup occludes part of the user's chest with respect to camera 1, but the missing surfaces are filled with data from camera 2.

3D Positional Error Table 1 shows the 3D positional error between cameras at two positions, measured by placing a $4 \mathrm{~cm}$ physical ball in the environment and measuring the difference in location between cameras. To determine the position of the ball, a virtual ball was moved over the physical one in $1 \mathrm{~mm}$ increments over each axis until it aligned as closely as possible. Cameras pairs not listed in the tables were not able to see the ball in a common position.

Our measured 3D positional error, which includes contributions from both camera calibration and depth sensor error, is fairly significant. However, since most of the depth cameras share the same general line of sight as would a remote participant, the perceived error contributed from depth sensor Z-error is reduced.

Table 1: 3D Error $(\mathrm{cm})$

\begin{tabular}{|lcc|}
\hline \multicolumn{3}{|c|}{ Accuracy near front of cubicle $(\approx 0.7 \mathrm{~m}$ from front cameras): } \\
& & \\
& Cam 2 (Front) & Cam 3 (Front) \\
\hline Cam 1 (Ceiling) & 1.86 & 3.03 \\
Cam 2 (Front) & - & 1.86 \\
\hline
\end{tabular}

\begin{tabular}{|lccc|}
\hline \multicolumn{4}{|c|}{ Accuracy near rear of cubicle $(\approx 1.8 \mathrm{~m}$ from front): } \\
& & & \\
& Cam 3 (Front) & Cam 4 (Rear) & Cam 5 (Rear) \\
\hline Cam 2 (Front) & 2.61 & 1.53 & 0.64 \\
Cam 3 (Front) & - & 3.63 & 2.71 \\
Cam 4 (Rear) & - & - & 1.75 \\
\hline
\end{tabular}

* Camera numbers correspond to labels in upper left of Figure 3

\subsection{Data Processing and Rendering Results}

Mesh Generation All images in Figure 8 show the result of mesh generation. Our requirements are met: the mesh offers a continuous surface, discontinuous surfaces (such as from the body to

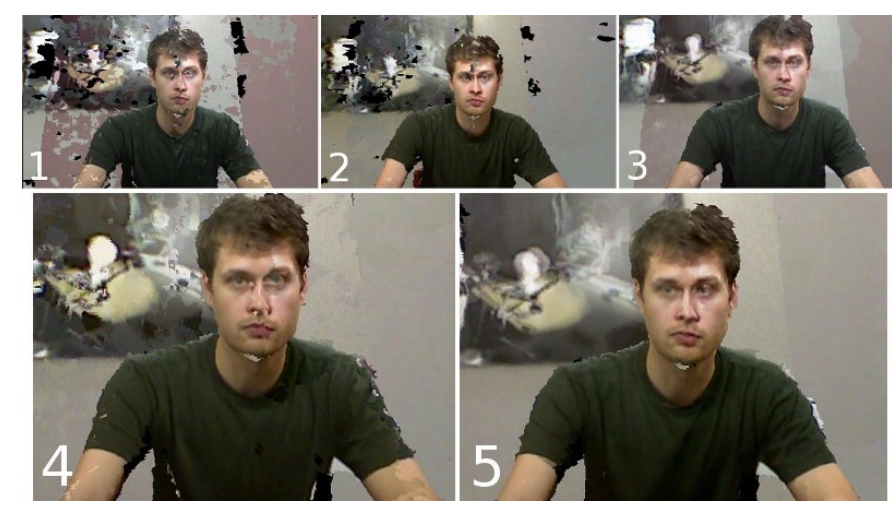

Figure 8: Data processing results. 1: No enhancements applied. 2: All enhancements except hole filling. 3: All enhancements except color matching. 4: All enhancements except quality weighted data merger. 5: All enhancements applied. ("All enhancements" includes to hole filling, data merger, and color matching)
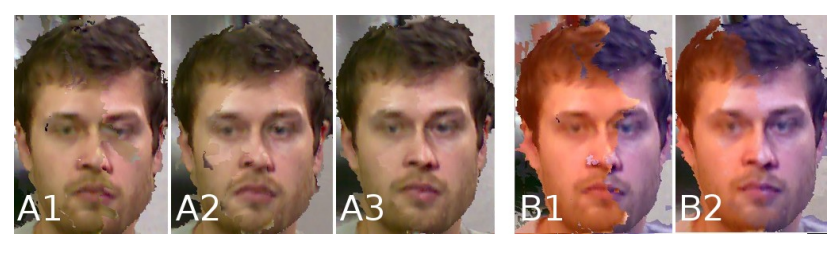

Figure 9: Data merger results. A1: No merger applied, meshes drawn on top of each other. A2: Merger with simple average. A3: Merger with quality weighting. B1: Color coded camera contributions with no merger. B2: Camera contributions with quality-weighted merger.

the rear wall) are properly separated, and missing data (small area under chin) is tolerated. An area for improvement is the edges of the mesh at discontinuous surfaces, which are ragged due to noise.

Hole Filling Image 5 of Figure 8 (as compared to image 2 of Figure 8) shows the result of the application of the hole filling and smoothing filter on a scene with four overlapping cameras. In this example, $100 \%$ of holes caused by interference were filled while textures remained aligned to the mesh (rates of $>90 \%$ are typical). The mesh edges were generally maintained, although a small amount of overfilling is present on the right shoulder and under the left arm causing the geometry to expand.

Data Merger Image 5 of Figure 8 (as compared to image 4 of Figure 8) and all of Figure 9 show the result of the data merger algorithm on four cameras, which is cleaner and smoother than meshes simply drawn over each other or averaged. In image B1 of Figure 9, one can see in the unmerged example that the mesh of the right-front camera (tinted blue) is drawn entirely over the data from the left-front camera (tinted red). These surfaces should coincide exactly, but a small calibration error places the surface from rightfront camera closer to the viewer. In image B2 of Figure 9, one can see that the quality-weighted merger algorithm smoothly transitions between camera data across the face.

Color Matching Image 5 of Figure 8 (as compared to image 3 of Figure 8) shows the result of color matching in a scene with four cameras. The unmodified image shows moderate color inconsistencies between the front cameras (on the face, shirt, and arms) and significant inconsistencies between the four cameras that overlap in the background. The automatic color matching algorithm mostly resolved the color deviations in the foreground, and made a significant improvement in color calibration the the background, although the camera coverage boundaries are still visible. 
Table 2: Tracking performance over 1600 frames.

\begin{tabular}{lccc} 
Case & $\#$ & $\%$ & Avg Time $(\mathrm{ms})$ \\
\hline Eyes found (full frame face/eye detect) & 3 & 0.19 & 140.5 \\
Eyes found (partial frame face/eye detect) & 9 & 0.56 & 19.8 \\
Eyes found (pattern search) & 1585 & 99.06 & 2.3 \\
Eyes not found & 3 & 0.19 & 13.6
\end{tabular}
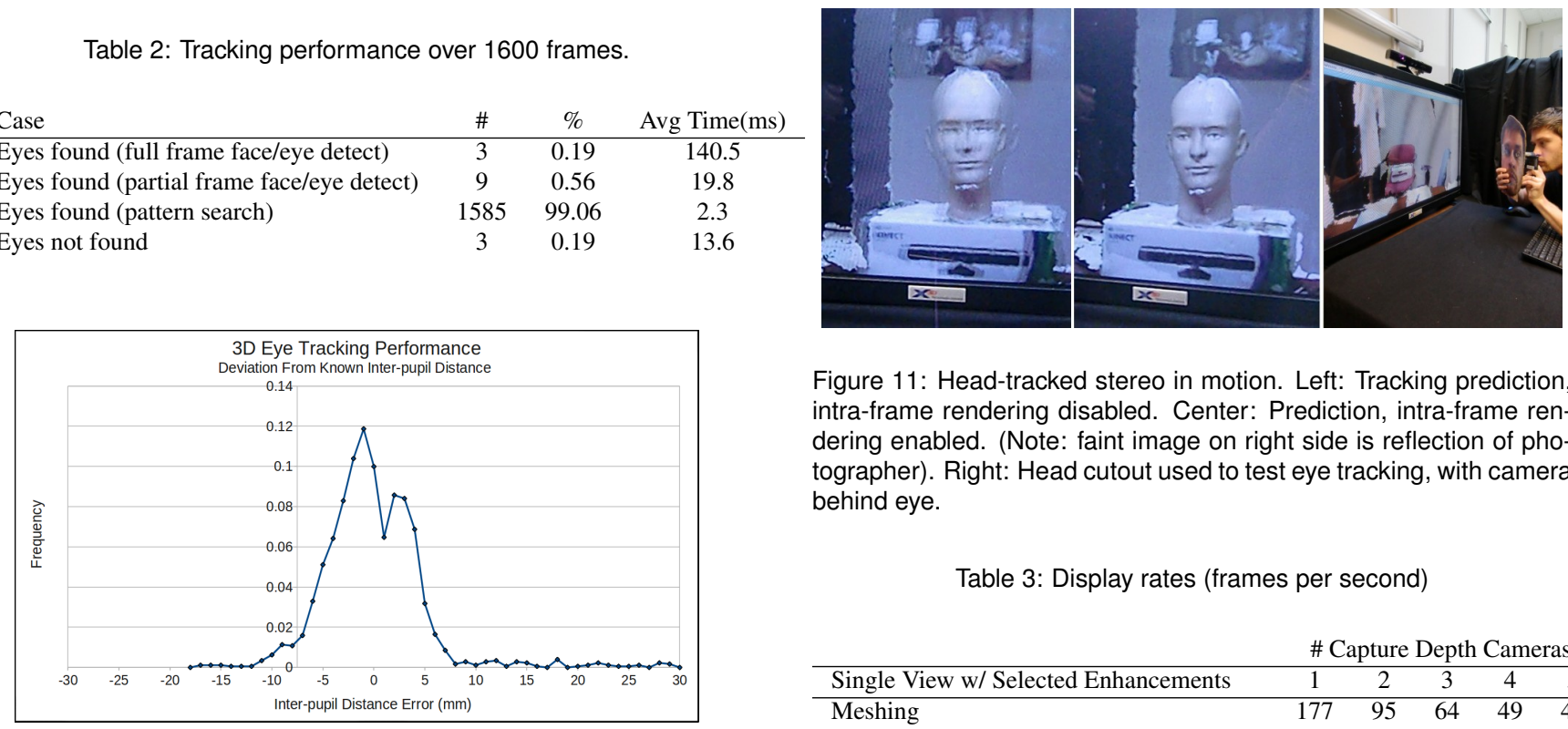

Figure 11: Head-tracked stereo in motion. Left: Tracking prediction, intra-frame rendering disabled. Center: Prediction, intra-frame rendering enabled. (Note: faint image on right side is reflection of photographer). Right: Head cutout used to test eye tracking, with camera behind eye.

Table 3: Display rates (frames per second)

Figure 10: 3D Eye tracking performance. Plot shows measured deviations from a known inter-pupil distance.

\subsection{Eye Tracking and Stereo Display Results}

Eye Detection Rate and Speed Table 2 shows the tracking performance typical of a seated user over a 1600 frame sequence. For the sequence, the user was seated centered $1 \mathrm{~m}$ from the display and tracking camera and moved his head left, right, forward and backward over a range of $\pm 0.5 \mathrm{~m}$. The average head movement speed was $48 \mathrm{~cm} / \mathrm{s}$, measured using the detected 3D eye positions. Positive eye detection occurred on $>99 \%$ of the frames at an average rate of $2.7 \mathrm{~ms}$. In the worst case, when the face was lost and the entire frame had to be searched, a noticeable delay of $140.5 \mathrm{~ms}$ on average occurred.

Tracking Performance Figure 10 provides a measure of the performance of the eye tracking by comparing the 3D distance between a pair of tracked eyes and the true measured interpupillary distance (IPD). IPD was used as the ground truth for accuracy as we were not in possession of equipment that would allow us to measure our positional accuracy directly. This metric was measured over a sequence of 1761 frames, in which a user seated $1 \mathrm{~m}$ from the tracking camera moved his head to the left, right, forward and backward over a range of $\pm 0.5 \mathrm{~m}$. $85.6 \%$ of measurements were within \pm 5 $\mathrm{mm}$ of the true IPD, and $96.4 \%$ were within $\pm 10 \mathrm{~mm}$.

Tracking Accuracy and Stereo Quality Since our tracking system is designed to support a stereo display, it is useful to test the two systems together. To demonstrate that our tracking system is fast and accurate enough to support our parallax barrier autostereo display with good quality, we shot video of our system through a tracking target (shown in the right of Figure 11). Our tracking system is able to detect the target as if it were a real face and thus one of the stereo views will be generated from the correct perspective of the camera placed behind an eye. Using this setup, the target and camera were positioned $1.25 \mathrm{~m}$ from the tracking camera and display and were moved at a rate of approximately $24 \mathrm{~cm} / \mathrm{sec}$.

Without tracking prediction and intra-frame rendering enabled, the rendering and parallax barrier pattern generation rate was 21 $\mathrm{Hz}$ in our four camera test setup. As seen in the left of Figure 11, results were very poor; the tracking and rendering could not keep up with the target as it moved into the viewing zone intended for the other eye and thus both views could be seen prominently and

simultaneously. With tracking prediction and intra-frame rendering enabled (center of Figure 11), the rendering rate dropped slightly to $18 \mathrm{~Hz}$ but the barrier generation rate more than doubled to 48 Hz. Results were much improved - the view seen by the camera is crisp and only very faint ghosting can be seen to the right of the mannequin head and box.

Gaze Preservation As can be seen in all images of Figure 8, the seated user whose gaze is directed forward appears to be looking directly at the remote user.

\subsection{System Performance}

Table 3 lists the performance achieved with our test system in various configurations. When rendering for a single view, the system was able to maintain frame rates $>30 \mathrm{~Hz}$ for up to five depth cameras with all enhancements (meshing, hole filling, quality-weighted data merger) enabled. For tracked stereo configurations, rendering rates fell to $>15 \mathrm{~Hz}$, but parallax barrier pattern rates of $\geq 48 \mathrm{~Hz}$ preserve smooth head tracking and stereo quality.

\section{Conclusions and FutURe Work}

We have presented solutions to several issues related to building a capture system using multiple depth cameras: resolving interference, data merging, and color matching between units. We have also introduced an eye position tracking system using depth sensors and demonstrated effective stereo display using rendering rates that would not usually support significant head motion.

Using these solutions, we have demonstrated a telepresence system that is able to capture a fully dynamic 3D scene the size of a cubicle while allowing a remote user to look around the scene from any viewpoint. The system preserves eye gaze and does not require the user to wear any encumbrances. Using a single PC and graphics card, our system was able to render head-tracked stereo views at interactive rates and maintained stereo percept even with moderate head movement speeds. 
Although our test system is functional, there are areas that we would like to improve, notably image quality. Our meshing approach left many captured objects with a ragged edge that could be smoothed with more advanced depth image filtering, such as a Joint Bilateral filtering [11] with the color image. Color calibration could be enhanced by combining our color correspondence-building algorithm with more robust color matching functions. Tracking support for faster head movement would also improve our user experience.

We also intend to expand our test setup into the "ideal" system shown in the bottom of Figure 3 by supporting 3D capture and 3D display for multiple users in both spaces. As seen in Figure 2, we already support 3D capture of multiple users. In this future system, we intend to add support for multiple tracked users on both sides, provide a larger display that emulates the large "virtual window" of [23], and support a larger office-sized capture area.

Finally, we would like to expand the communication ability of our system by adding support for virtual objects that can be manipulated naturally by persons in the scene. Figure 1E shows an early experiment.

\section{ACKNOWLEDGEMENTS}

The authors would like to thank Herman Towles for proposing this system, Andrei State for helping to produce the supplemental video and both for making helpful suggestions for improving this paper. We also thank Kurtis Keller for advice on reducing Kinect interference, Adrian Ilie for recommendations regarding color calibration, Ava Pope for helping to measure the wavelength of the Kinect's IR lasers and Jonathan Bidwell for technical discussions and advice. This work was supported in part by the National Science Foundation (award CNS-0751187) and by the BeingThere Centre, a collaboration of UNC Chapel Hill, ETH Zurich, NTU Singapore, and the Media Development Authority of Singapore.

\section{References}

[1] H. H. Baker, N. Bhatti, D. Tanguay, I. Sobel, D. Gelb, M. E. Goss, W. B. Culbertson, and T. Malzbender. Understanding performance in coliseum, an immersive videoconferencing system. ACM Trans. Multimedia Comput. Commun. Appl., 1:190-210, May 2005.

[2] T. Balogh and P. T. Kovács. Real-time 3d light field transmission. volume 7724, page 772406. SPIE, 2010.

[3] Q. Cai, D. Gallup, C. Zhang, and Z. Zhang. 3d deformable face tracking with a commodity depth camera. In Proceedings of the 11th European conference on computer vision conference on Computer vision: Part III, ECCV'10, pages 229-242, Berlin, Heidelberg, 2010. Springer-Verlag.

[4] M. Castrillón Santana, O. Déniz Suárez, M. Hernández Tejera, and C. Guerra Artal. Encara2: Real-time detection of multiple faces at different resolutions in video streams. Journal of Visual Communication and Image Representation, pages 130-140, April 2007.

[5] M. A. Fischler and R. C. Bolles. Random sample consensus: a paradigm for model fitting with applications to image analysis and automated cartography. Commun. ACM, 24:381-395, June 1981.

[6] B. FREEDMAN, A. SHPUNT, M. MACHLINE, and Y. ARIELI. Depth mapping using projected patterns. Patent Application, 052010. US 2010/0118123 A1.

[7] M. Gross, S. Würmlin, M. Naef, E. Lamboray, C. Spagno, A. Kunz, E. Koller-Meier, T. Svoboda, L. Van Gool, S. Lang, K. Strehlke, A. V. Moere, and O. Staadt. blue-c: a spatially immersive display and $3 \mathrm{~d}$ video portal for telepresence. ACM Trans. Graph., 22:819-827, July 2003.

[8] A. Ilie and G. Welch. Ensuring color consistency across multiple cameras. In Proceedings of the Tenth IEEE International Conference on Computer Vision - Volume 2, ICCV '05, pages 1268-1275, Washington, DC, USA, 2005. IEEE Computer Society.

[9] A. Jones, M. Lang, G. Fyffe, X. Yu, J. Busch, I. McDowall, M. Bolas, and P. Debevec. Achieving eye contact in a one-to-many $3 \mathrm{~d}$ video teleconferencing system. ACM Trans. Graph., 28:64:1-64:8, July 2009.
[10] H. Kawata and T. Kanai. Image-based point rendering for multiple range images. In Proceedings of the 2nd International Conference on Information Technology for Application (ICITA 2004), pages 478 -483, Sydney, January 2004. Macquarie Scientific Publishing.

[11] J. Kopf, M. F. Cohen, D. Lischinski, and M. Uyttendaele. Joint bilateral upsampling. ACM Trans. Graph., 26, July 2007.

[12] G. Kurillo, R. Bajcsy, K. Nahrsted, and O. Kreylos. Immersive 3d environment for remote collaboration and training of physical activities. In Virtual Reality Conference, 2008. VR '08. IEEE, pages $269-270$, march 2008.

[13] R. Lienhart and J. Maydt. An extended set of haar-like features for rapid object detection. In Image Processing. 2002. Proceedings. 2002 International Conference on, volume 1, pages I-900 - I-903 vol.1, 2002.

[14] W. Matusik and H. Pfister. 3d tv: a scalable system for real-time acquisition, transmission, and autostereoscopic display of dynamic scenes. ACM Trans. Graph., 23:814-824, August 2004.

[15] M. McGuire. A fast, small-radius gpu median filter. In ShaderX6, February 2008.

[16] M. Meehan. Physiological Reaction as an Objective Measure of Presence in Virtual Environments. PhD thesis, University of North Carolina at Chapel Hill, 2001.

[17] P. Merrell, A. Akbarzadeh, L. Wang, P. Mordohai, J.-M. Frahm, R. Yang, D. Nister, and M. Pollefeys. Real-time visibility-based fusion of depth maps. In Computer Vision, 2007. ICCV 2007. IEEE 11th International Conference on, pages 1-8, oct. 2007.

[18] L. Muhlbach, M. Bocker, and A. Prussog. Telepresence in videocommunications: a study on stereoscopy and individual eye contact. Human Factors, 37:16, June 1995.

[19] T. Peterka, R. Kooima, J. Girado, J. Ge, D. Sandin, A. Johnson, J. Leigh, J. Schulze, and T. DeFanti. Dynallax: Solid state dynamic parallax barrier autostereoscopic vr display. In Virtual Reality Conference, 2007. VR' '07. IEEE, pages $155-162$, march 2007.

[20] B. Petit, J.-D. Lesage, C. Menier, J. Allard, J.-S. Franco, B. Raffin, E. Boyer, and F. Faure. Multicamera real-time 3d modeling for telepresence and remote collaboration. INTERNATIONAL JOURNAL OF DIGITAL MULTIMEDIA BROADCASTING, 2010:247108-12, 2009.

[21] R. Raskar, G. Welch, M. Cutts, A. Lake, L. Stesin, and H. Fuchs. The office of the future: a unified approach to image-based modeling and spatially immersive displays. In Proceedings of the 25th annual conference on Computer graphics and interactive techniques, SIGGRAPH '98, pages 179-188, New York, NY, USA, 1998. ACM.

[22] O. Schreer, I. Feldmann, N. Atzpadin, P. Eisert, P. Kauff, and H. Belt. 3dpresence -a system concept for multi-user and multi-party immersive 3d videoconferencing. In Visual Media Production (CVMP 2008), 5th European Conference on, pages 1 -8, nov. 2008.

[23] H. Towles, W.-C. Chen, R. Yang, S.-U. Kum, H. F. N. Kelshikar, J. Mulligan, K. Daniilidis, H. Fuchs, C. C. Hill, N. K. J. Mulligan, L. Holden, B. Zeleznik, A. Sadagic, and J. Lanier. 3d telecollaboration over internet2. In International Workshop on Immersive Telepresence, Juan Les Pins, 2002.

[24] P. Viola and M. Jones. Rapid object detection using a boosted cascade of simple features. In Computer Vision and Pattern Recognition, 2001. CVPR 2001. Proceedings of the 2001 IEEE Computer Society Conference on, volume 1, pages I-511 - I-518 vol.1, 2001.

[25] G. Ye, A. State, and H. Fuchs. A practical multi-viewer tabletop autostereoscopic display. In Mixed and Augmented Reality (ISMAR), 2010 9th IEEE International Symposium on, pages 147 -156, oct. 2010.

[26] Z. Zhang. Flexible camera calibration by viewing a plane from unknown orientations. In Computer Vision, 1999. The Proceedings of the Seventh IEEE International Conference on, volume 1, pages 666 -673 vol.1, 1999. 